\title{
Response Element
}

National Cancer Institute

\section{Source}

National Cancer Institute. Response Element. NCI Thesaurus. Code C13372.

A nucleotide sequence that plays a role in the expression of a nearby gene because it can be recognized by specific transcription factors or coregulatory proteins. 